\title{
Analysis of Gene Expression in 4,4'-Methylenedianiline-induced Acute Hepatotoxicity
}

\author{
Jung-Hwa Oh ${ }^{1}$, Hea-Jin Yoon ${ }^{1}$, Jung-Sun Lim ${ }^{1}$, Han-Jin Park', Jae-Woo Cho ${ }^{2}$, \\ Myung-Sang Kwon ${ }^{3,4}$ and Seokjoo Yoon ${ }^{1}$ \\ ${ }^{1}$ Toxicogenomics Team, ${ }^{2}$ Clinical Pathology Team, \\ ${ }^{3}$ Korea Institute of Toxicology, 19 Shinsung-ro, Yuseoung, Daejeon 305-343 \\ ${ }^{4}$ School of Veterinary Medicine, Kangwon National University, Chuncheon 200-701, Korea
}

(Received February 25, 2009; Revised May 13, 2009; Accepted May 13, 2009)

\begin{abstract}
4,4'-Methylenedianiline (MDA) is an aromatic amine that is widely used in the industrial synthetic process. Genotoxic MDA forms DNA adducts in the liver and is known to induce liver damage in human and rats. To elucidate the molecular mechanisms associated with MDA-induced hepatotoxicity, we have identified genes differentially expressed by microarray approach. BALB/c male mice were treated once daily with MDA $(20 \mathrm{mg} / \mathrm{kg})$ up to 7 days via intraperitoneal injection (i.p.) and hepatic damages were revealed by histopathological observation and elevation of serum marker enzymes such as AST, ALT, ALP, cholesterol, DBIL, and TBIL. Microarray analysis showed that 952 genes were differentially expressed in the liver of MDA-treated mice and their biological functions and canonical pathways were further analyzed using Ingenuity Pathways Analysis (IPA). Toxicological functional analysis showed that genes related to hepatotoxicity such hyperplasia/hyperproliferation (Timp1), necrosis/cell death (Cd14, Mt1f, Timp1, and Pmaip1), hemorrhaging (Mt1f), cholestasis (Akr1c3, Hpx, and S/c10a2), and inflammation (Cd14 and Hpx) were differentially expressed in MDA-treated group. This gene expression profiling should be useful for elucidating the genetic events associated with aromatic amine-induced hepatotoxicity and for discovering the potential biomarkers for hepatotoxicity.
\end{abstract}

Key words:4,4'-Methylenedianiline, Hepatotoxicity, Gene expression profiling

\section{INTRODUCTION}

4,4'-Methylenedianiline (MDA) is a primary aromatic amine used extensively in the manufacture of polyamides, epoxy resins and polymers in variety of polyurethane industry (Mcqueen and Williams, 1990). MDA is an important intermediate in the production of 4,4'-methylenediphenyl diisocyanate (MDI), and also used in the plastic industries (Zhang et al., 2006). Because of widespread use and large scale production of both MDI and MDA, the potential risk for human exposure and intoxication from MDA is significant. Humans exposed to MDA accidentally or occupationally develop injuries to cholangiocytes with subsequent cholestasis, cholangitis, jaundice, toxic hepatitis with elevated serum liver enzymes (Bastian, 1984; Nichols, 2004). Studies in rats

Correspondence to: Seokjoo Yoon, Toxicogenomics Team, Korea Institute of Toxicology, 19 Shinsung-ro, Yuseoung, Daejeon, 305-343, Korea

E-mail: sjyoon@kitox.re.kr have shown that moderate doses of MDA induce a dose- and time-dependent cholestasis, rapid and severe injury to biliary epithelial cells and moderate hepatotoxicity (Santa Cruz et al., 2007). To date, MDA is known to be genotoxic by forming DNA adducts in the liver and induce the DNA damage in rat or human hepatocytes (Martelli et al., 2002; McQueen and Williams, 1990; Schutze et al., 1995). In addition, N-Acetyl MDA has been identified as a major metabolite (in rat and human urine) of MDA or MDI (Robert et al., 1995; Schutze et al., 1995). N-Acetyl MDA which is catalyzed by $\mathrm{N}$-acetyltransferase 1 (NAT1) and 2 (NAT2) has been known to be an important factor for susceptibility toward MDA-induced hepatotoxicity (Zang et al., 2005). However, little is known about its molecular mechanism of MDA-induced hepatotoxicity, although studies about acute MDA exposure indicated that biliary epithelial cells of the liver and common bile duct are the early site of injury.

Microarray analysis have been applied extensively to elucidate the molecular mechanisms underlying hepato- 
toxicity (Heijne et al., 2004; Huang et al., 2004; Oh et al., 2007), nephrotoxicity (Oh et al., 2006), and other toxicological phenomena (Oh et al., 2009a, b) and to alternatively assess toxicity (Hamadeh et al., 2002). In the present study, gene expression profiling of MDAinduced hepatotoxicity was analyzed using microarray approach to identify the genes associated with liver injury and understand the mechanism of MDA-induced hepatotoxicity in molecular level. Gene expression profiling was here analyzed in mice after administration of MDA across various time points using $10 \mathrm{~K}$ Mouse oligo chip, supported by histopathology observation of the liver and biochemical analysis.

\section{MATERIALS AND METHODS}

Animal and chemical. Methylenedianiline (MDA) was purchased from Sigma, and approximately 9-weekold BALB/c male mice (SLC, Inc., Japan) were kept in a 12-h light/dark cycle, under controlled temperature and humidity for 2 weeks prior to experiment in the animal room. The mice were fed standard food pellets. MDA was dissolved in $30 \%$ polyethylene glycol and was administered once daily at $20 \mathrm{mg} / \mathrm{kg}$ up to 7 day via intraperitoneal injection (i.p.). Five mice were used in each test group and necropsies were then performed at 1,3 and 7 day.

Biochemical analysis. Blood was collected from the inferior vena cava. Serum was separated by centrifugation. Alanine aminotransferase (ALT), aspartate aminotransferase (AST), alkaline phosphatase (ALP) activities, cholesterol, direct bilirubin (DBIL), and total bilirubin (TBIL) were determined using an automated clinical chemistry analyzer (Fuji Dri-Chem 3500s, Fujifim Co., Japan), at each time point. Data are expressed as the mean \pm S.D. of 3 selected samples with hiotopathological lesion. The statistical significance between various experimental groups was tested using one-way ANOVA followed by the Dunnett test. $P$-values less than 0.05 were considered to be significant.

Histopathology. Liver samples collected from treated and control mice were fixed in $10 \%$ neutral buffered formalin, and embedded in paraffin. The sections ( $4 \mu \mathrm{m}$ thick) were cut using RM2165 microtome (Leica, Germany), stained with hematoxylin and eosin (H\&E), and examined under a light microscope (Nikon E400, Nikon, Japan).

Isolation of total RNA. The left lateral lobe of the liver was removed and processed for RNA extraction. For microarray analysis, total RNA was extracted using the TRIzol reagent (Invitrogen, USA) according to the instructions of the manufacturer and purified using $\mathrm{RNe}-$ asy total RNA isolation kit (QIAGEN, Germany). Total RNA was quantified by NanoDrop ND-1000 (NanoDrop, USA) and its integrity was assessed by 2100 Bioanalyzer (Agilent Technologies, USA).

Microarray analysis. For microarray analysis, fluorescent-labeled CDNA was prepared by reverse-transcription of total RNA in the presence of the coupled Cy3-dUTP or Cy5-dUTP (NEN) using Superscript II (Invitrogen, USA). Single-stranded cDNA probes were purified using a PCR purification kit (QIAGEN, Germany). Probes were re-suspended in hybridization solution ( $50 \%$ formamide, $5 \times$ SSC, $0.1 \%$ SDS). The $10 \mathrm{~K}$ Mouse oligo chip (GenomicTree, Korea) was hybridized with the fluorescent-labeled cDNAs at $42^{\circ} \mathrm{C}$ in a humid chamber for 16 hours. After proper washing, the slides were scanned using Axon GenePix 4000B (Axon Instrument, USA). Scanned images were calculated with GenePix 3.0 software (Axon Instrument, USA) and analyzed with Genplex ver. 2.0 software (Istech, Korea). Background of spot intensity was corrected and miscellaneous spot were removed. The log gene expression ratios were normalized by Block-wise centering (Print-tip Lowess normalization) method. The differentially expressed genes were selected based on 2-fold changes in all of the treated groups compared with the levels in the pooled controls on each time point $(1,3$, and 7 days). Totally, 952 differentially expression genes were selected and then clustering analysis were performed using $k$-means partitioning clustering based on Euclidean distances $(k=6)$. The biofunctional and canonical pathway analysis of differentially expressed genes was performed using Ingenuity Pathways Analysis (IPA). Selected genes were annotated based on Probe ID with NCBI Genbank accession number.

\section{RESULTS}

Biochemical evaluation. The enzyme activities relative to liver injuries were significantly changed in all time points between MDA-treated and control group (Fig. 1). After $20 \mathrm{mg} / \mathrm{kg} /$ day MDA treatment, AST which is the index of acute phase liver toxicity was elevated most in day 1 and chronic phase liver enzyme ALT was markedly increased in day 7. ALP and cholesterol activities were also increased in day 3 and day 7 . Serum level of direct bilirubin (DBIL) and total bilirubin (TBIL) were all increased in MDA-treated group compared to control group but the value of treated group decreased time dependent manner. 
(A)

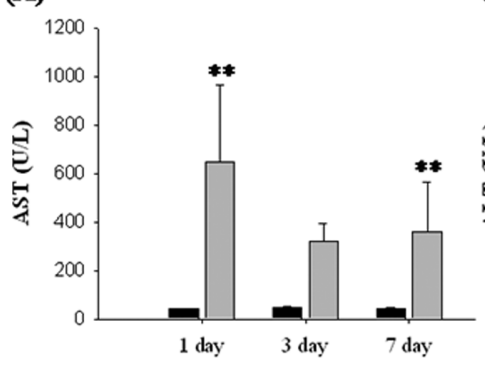

(D)

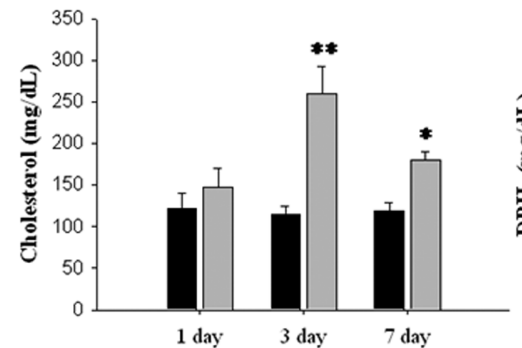

(B)

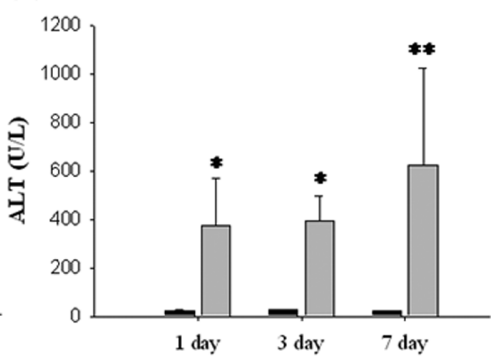

(E)

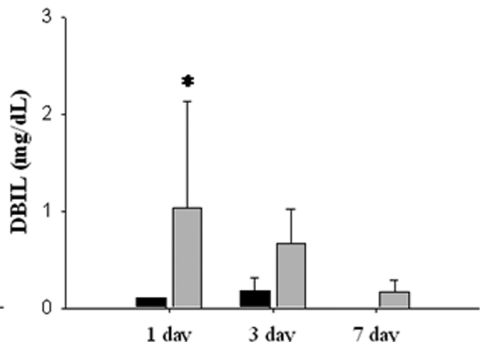

(C)

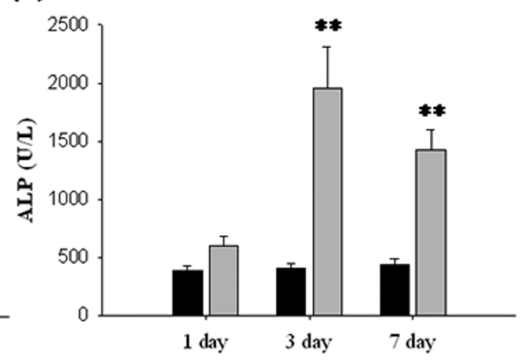

(F)

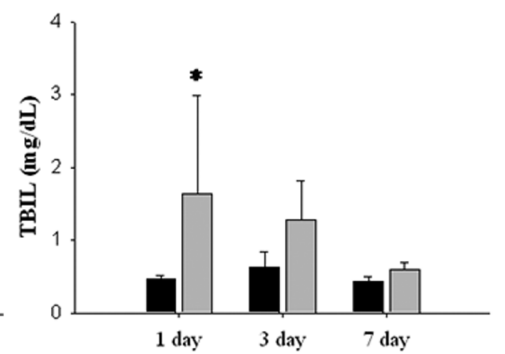

Fig. 1. Blood biochemical data in the liver of MDA treated mice. (A) AST (aspartate aminotransferase); (B) ALT (alanine aminotransferase); (C) ALP (alkaline phosphatase); (D) Cholesterol; (E) DBIL (direct bilirubin); (F) TBIL (total bilirubin). Values represent the means \pm standard deviation (SD); $\mathrm{n}=3\left({ }^{*} P<0.05 ;{ }^{* *} P<0.01\right)$.

Table 1. Summary of histophathological lesions in the liver

\begin{tabular}{lcccc}
\hline \hline & Control & & MDA-treated group \\
\cline { 3 - 4 } & $(\mathrm{n}=5)$ & 1 Day $(\mathrm{n}=3)$ & 3 Day $(\mathrm{n}=3)$ & 7 Day $(\mathrm{n}=3)$ \\
\hline Inflammatory foci & $+(3)$ & & $+(2)$ & $+(3)$ \\
Periductular inflammation & & $++(2)+++(1)$ & $++(2)+++(1)$ \\
Necrosis/congestion, hepatocyte & $+(1)++(1)+++(1)$ & $++(2)+++(1)$ & $++(1)+++(2)$ \\
Mitosis & & $++(1)$ & $+(1)$ \\
Ductular hyperplasia & & $+(1)$ & $++(2)$ \\
Hepatocellular vacuolation, centrilobular & & & $++(1)$ \\
Regenerative inflammation & & & $+(1)$ \\
\hline
\end{tabular}

The number of animal with corresponding lesion was represented in the parentheses.

Severity of lesion was indicated as follows; +, mild; ++, medium; +++, severe.

Histopathology. Hepatocytes near the central vein regions of MDA treated mice were compared. Light microscopy showed a decreased in the number of hepatocyte and increased in the size compared to the central vein region of control mice. Periductular inflammation and necrosis/congestion of the hepatocytes were observed in all time points along with the severity of the lesions increased. MDA treatment for 7 days resulted in medium ductular hyperplasia and regenerative inflammation (Table 1).

Gene expression profiles of MDA-induced hepatotoxicity. Microarray analysis was carried out to elucidate the molecular mechanism of hepatic gene expression between vehicle- (30\% polyethylene glycol) and MDA-treated mice. Differentially expressed genes in the
MDA-treated group were selected based on changes greater than 2-fold compared with the corresponding controls, in all independent experiment. Totally, 952 genes were differentially expressed, including 149,454 , and 577 genes at day 1 , day 3 , and day 7 in MDAtreated group. To analyze the gene expression patterns according to each time points during MDA-induced hepatotoxicity, total 952 genes were processed in clustering analysis using $k$-means partitioning clustering (Fig. 2). Clustering analysis showed that many genes were down-regulated in the MDA-treated group although individual variation was shown. In particular, many genes belonged to down-regulated clusters were upregulated at day 3 . However, we focused on commonly up- or down-regulated genes in MDA-treated group such as Cluster $A$ and $B$, respectively. In all time points, 

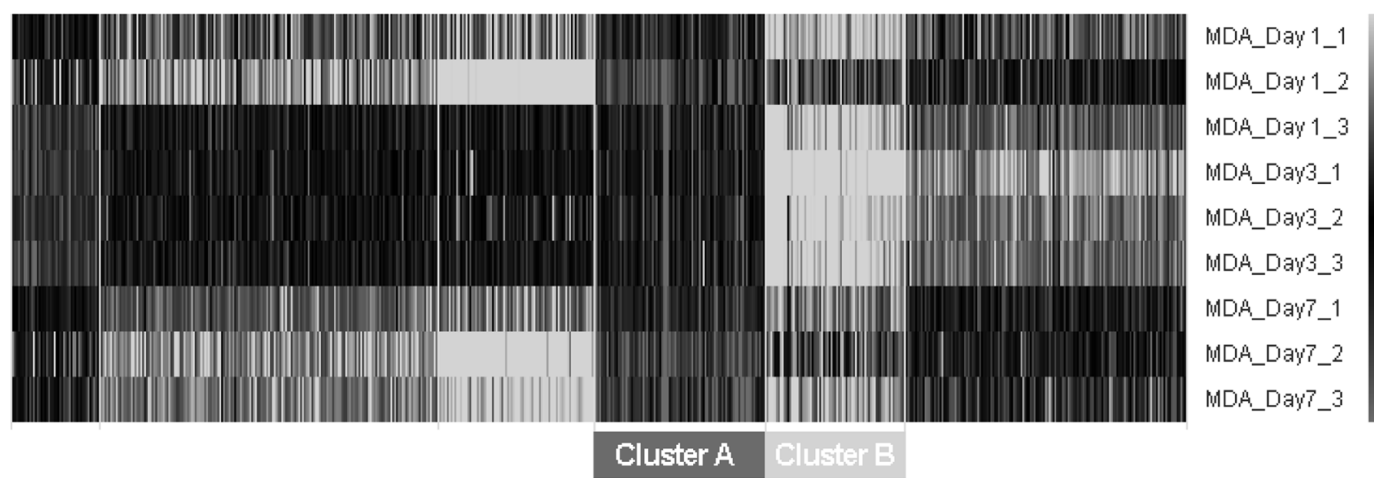

Cluster $A$

Fig. 2. The $k$-means clustering of differentially expressed genes in the liver of MDA treated mice. 952 genes were analyzed based on 2 -fold changes and we performed $k$-means clustering by setting $k=6$ (total clusters $=6$ ). The six clusters are highlighted with colored lines. Cluster A and cluster B indicates the commonly up- or down-regulated group at all time points.

140 genes were significantly up-regulated, while 112 genes were down-regulated in MDA-treated group.

From among the genes up- or down-regulated in common, the top 20 genes that were significantly altered in MDA-treated group were selected and are listed in Table 2 and Table 3. Genes encoding growth factor such as Gdf15 and Gdnf, and several genes encoding various transporters or receptors such as Fcer1g, C3ar1, Pgm1, Sftpd, and Saa3 were up-regulated. In addition, genes related to inflammatory response (Ccl24 and Itih3), regulation of transcription or proliferation (Hic1 and Slfn1), signal transduction (Apbb1ip and
Ltb4r1), and leukocyte chemotaxis or cytoskeleton reorganization (S100a9) were up-regulated. On the other hand, genes such as Pramef12 and Pr15a8 involved in hormone activity, and ion channel or transport such as Kcmf1, 5330439B14Rik, and Slc4a4, were down-regulated. In addition, many genes related to metabolic process (Plb1, Dgat1, Naaa, Gk2, and Dhah) were downregulated.

Toxicological interpretation of differentially expressed genes during MDA-induced hepatotoxicity. To investigate the molecular mechanism related to

Table 2. Genes up-regulated in the liver of MDA-treated mice

\begin{tabular}{|c|c|c|c|c|c|}
\hline \multirow{2}{*}{$\begin{array}{l}\text { Gene } \\
\text { symbol }\end{array}$} & \multirow{2}{*}{ Gene title } & \multirow{2}{*}{ Acc. no. } & \multicolumn{3}{|c|}{ Fold Change (Log2) } \\
\hline & & & Day 1 & Day 3 & Day 7 \\
\hline Gdf15 & growth differentiation factor 15 & NM_011315 & 0.60 & 3.02 & 4.33 \\
\hline Flot2 & flotillin 2 & $\mathrm{~L} 47 \overline{6} 96$ & 3.86 & 5.11 & 4.30 \\
\hline Fcer1g & Fc receptor, IgE, high affinity I, gamma polypeptide & NM_009265 & 1.16 & 1.57 & 4.13 \\
\hline C3ar1 & complement component $3 a$ receptor 1 & NM_0ō_09114 & 3.83 & 1.34 & 3.94 \\
\hline $\mathrm{Cc} / 24$ & chemokine (C-C motif) ligand 24 & NM_016701 & 2.31 & 2.21 & 3.92 \\
\hline Pgm1 & phosphoglucomutase 1 & AK0̄02981 & 2.89 & 4.36 & 3.76 \\
\hline Sftpd & surfactant associated protein D & NM_008605 & 2.25 & 3.26 & 3.38 \\
\hline Hic1 & hypermethylated in cancer 1 & NM_009705 & 1.73 & 0.06 & 3.26 \\
\hline Slfn1 & schlafen 1 & NM_010766 & 0.41 & 1.53 & 3.19 \\
\hline Apbb1ip & amyloid beta (A4) precursor protein-binding, family $B$, member 1 interacting protein & AK018561 & 1.93 & 1.17 & 3.03 \\
\hline Gdnf & glial cell line derived neurotrophic factor & ВC003991 & 1.18 & 1.62 & 2.89 \\
\hline Cd14 & CD14 antigen & NM_009160 & 1.42 & 0.73 & 2.87 \\
\hline Itih3 & inter-alpha trypsin inhibitor, heavy chain 3 & NM_007737 & 1.07 & 2.03 & 2.76 \\
\hline Yif1b & Yip1 interacting factor homolog B (S. cerevisiae) & AKŌ07508 & 2.20 & 0.81 & 2.74 \\
\hline$L t b 4 r 1$ & leukotriene B4 receptor 1 & NM_007743 & 0.73 & 1.62 & 2.39 \\
\hline Mrps28 & mitochondrial ribosomal protein S28 & AK0̄10014 & 1.67 & 3.17 & 2.38 \\
\hline S100a9 & S100 calcium binding protein A9 (calgranulin B) & NM_008567 & 2.99 & 4.46 & 2.37 \\
\hline$C d k / 2$ & cyclin-dependent kinase-like 2 (CDC2-related kinase) & NM_013710 & 0.98 & 0.36 & 2.31 \\
\hline Saa3 & serum amyloid A 3 & NM_010662 & 2.06 & 2.22 & 2.31 \\
\hline$M s 4 a 4 b$ & membrane-spanning 4-domains, subfamily $A$, member $4 B$ & NM_023380 & 1.26 & 0.42 & 2.28 \\
\hline
\end{tabular}

The fold change was calculated as the relative average value of three arrays in each group compared to controls, and the values are reported as the natural logarithm. 
Table 3. Genes down-regulated in the liver of MDA treated mice

\begin{tabular}{|c|c|c|c|c|c|}
\hline \multirow{2}{*}{$\begin{array}{l}\text { Gene } \\
\text { symbol }\end{array}$} & \multirow{2}{*}{ Gene title } & \multirow{2}{*}{ Acc. no. } & \multicolumn{3}{|c|}{ Fold Change (Log2) } \\
\hline & & & Day 1 & Day 3 & Day 7 \\
\hline Pramef12 & PRAME family member 12 & AK021319 & -3.52 & -4.79 & -4.32 \\
\hline Kcmf1 & potassium channel modulatory factor 1 & NM_019715 & -2.97 & -3.96 & -2.95 \\
\hline 1700067 C01Rik & RIKEN cDNA $1700067 \mathrm{C} 01$ gene & AK006911 & -3.31 & -4.75 & -2.89 \\
\hline Phb & prohibitin & AF061744 & -2.78 & -3.91 & -2.80 \\
\hline 5330439B14Rik & RIKEN cDNA 5330439B14 gene & AK019938 & -2.28 & -2.70 & -2.73 \\
\hline Pln & phospholamban & NM 023129 & -3.31 & -3.58 & -2.66 \\
\hline Plb1 & phospholipase B1 & AK019494 & -3.03 & -4.13 & -2.63 \\
\hline Slc4a4 & solute carrier family 4 (anion exchanger), member 4 & AF141934 & -2.59 & -3.57 & -2.59 \\
\hline Dgat1 & diacylglycerol O-acyltransferase 1 & NM 010046 & -2.11 & -2.70 & -2.50 \\
\hline 4921521F21Rik & RIKEN cDNA $4921521 F 21$ gene & NM_027582 & -2.34 & -3.10 & -2.50 \\
\hline Sfrp5 & secreted frizzled-related sequence protein 5 & NM_018780 & -3.04 & -4.01 & -2.42 \\
\hline Tnfsf8 & tumor necrosis factor (ligand) superfamily, member 8 & NM_009403 & -2.74 & -4.25 & -2.41 \\
\hline Sepw1 & selenoprotein $\mathrm{W}$, muscle 1 & NM_007770 & -2.67 & -3.89 & -2.38 \\
\hline Prl8a8 & prolactin family 8 , subfamily a, member 81 & AK005568 & -2.59 & -3.28 & -2.32 \\
\hline Gabra3 & gamma-aminobutyric acid (GABA-A) receptor, subunit alpha 3 & NM 008067 & -2.53 & -3.44 & -2.25 \\
\hline Zfp354c & zinc finger protein $354 \mathrm{C}$ & NM_013922 & -2.33 & -3.81 & -2.22 \\
\hline Tex18 & testis expressed gene 18 & NM_031385 & -2.99 & -3.65 & -2.21 \\
\hline Naaa & $\mathrm{N}$-acylethanolamine acid amidase & AK008776 & -1.88 & -2.63 & -2.18 \\
\hline Gk2 & glycerol kinase 2 & NM_010294 & -2.26 & -2.92 & -2.18 \\
\hline$D h d h$ & dihydrodiol dehydrogenase (dimeric) & AK005050 & -2.80 & -2.07 & -2.00 \\
\hline
\end{tabular}

The fold change was calculated as the relative average value of three arrays in each group compared to controls, and the values are reported as the natural logarithm.

MDA-induced hepatotoxicity, canonical pathways and toxicological pathways integrating differentially expressed genes were analyzed using IPA. Total 252 commonly deregulated genes at all time points including 140 upregulated and 112 down-regulated genes were characterized. As shown in Fig. 3A, down-regulated genes involved in GABA receptor signaling (Gabra1, Gabra2, and Gabra3), C21-steroid hormone metabolism (Akr1c3 and $H s d 3 b 4$ ) were identified. Meanwhile, expression of genes related to hepatic fibrosis/hepatic stellate cell activation (Ccr7, Col1a2, Cd14, and Timp1 for up-regulated, Fgf1d for down-regulated), leukocyte extravasation signaling (Arhgap12, Cldn7, Itgam, Mmp12, and Timp1 for up-regulated; Jam2 for down-regulated), eicosanoid signaling (Akrk3 and Ptger3 for up-regulated; Ltb3r for down-regulated), and glycosaminoglycan degradation (Glb1l, Hexb for up-regulated; Hyal4 for down-regulated) was altered.

The toxicological pathway analysis of differentially expressed genes showed that several toxic mechanism were identified and top ranked mechanisms were selected as shown Fig. 3B. Genes related to positive acute phase response proteins (Hpx and Saa1), hepatic stellate cell activation (Cd14 and Timp1), negative acute phase response proteins (Itih3), cholesterol biosynthesis (Sqle), LXR/RXR activation (Arg2 and Cd14) were up-regulated, while genes associated with cytochrome P450 panel (Cyp4a14), and anti-apoptosis (Bc/2/10) were down-regulated. In addition, genes related to hepatic fibrosis (Col1as, Sparc, and Timp1 for up-regulated; Fgf1 for down-regulated), FXR/RXR activation (Pck2 and Slc10a2 for up-regulated; C6pc2 for downregulated) were identified. Functional analysis using IPA also identified genes involved in hepatotoxicity such as liver hyperplasia/hyperproliferation, liver necrosis/cell death, liver hemorrhaging, liver cholestasis, and liver inflammation.

\section{DISCUSSION}

Exposure to MDA has been known to induce liver damages and an industrial outbreak of occupational MDA exposure provokes the public health concerns. Using toxicogenomic approaches, toxicological mechanisms and drug safety have been recently investigated. Several studies have shown that the prediction of toxicity is possible by using toxicogenomics, especially hepatotoxicity. To evaluate the molecular mechanisms of acute hepatotoxicity and to identify the molecular targets participating in these processes, we examined the effects of exposure to MDA on gene expression changes in the mice liver.

In the present study, histopathological observation of MDA-treated mice showed that severe hepatic lesions were detected in all time points such as necrosis of hepatocytes. In addition, blood biochemistry showed 
(A)

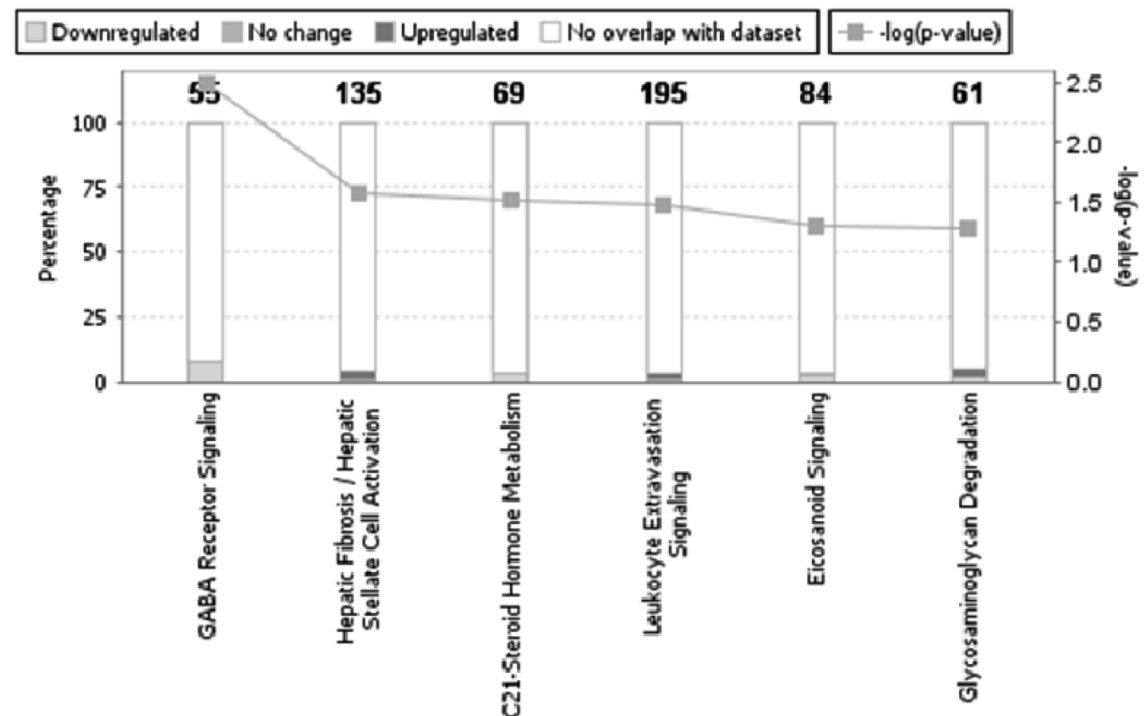

(B)

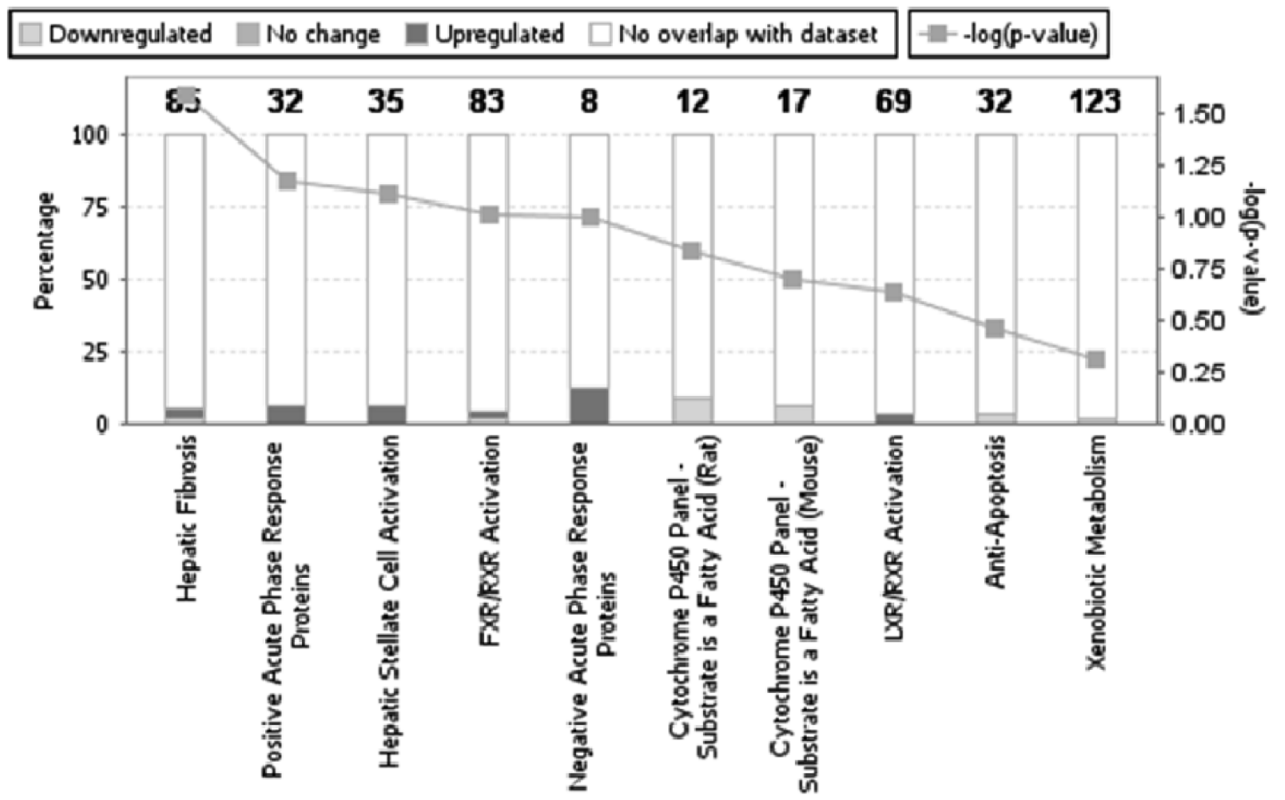

Fig. 3. Canonical pathway and toxicological analysis of 252 genes deregulated in MDA-treated group. (A) Canonical pathways which are identified based on statistical significance $(P<0.05)$. (B) Functional analysis showing categories of interest. Arabic numbers upon histogram indicates total number of genes belonged to designated pathways and colored box indicated up- or down-regulated genes which were identified in MDA-treated group as follows; red ones, up-regulated; green ones; down-regulated. Orange line indicates the statistical significance calculated by IPA algorithm, and values means natural logarithm (Log10).

that AST and ALT were significantly increased over at least 7-fold or 15-fold comparing to controls. These results indicate that MDA-induced toxicity has not occurred in a time-dependent manner however severe acute hepatotoxicity was shown in all time points after MDA administrations. Based on k-means clustering, microarray analysis also represented that gene expression profiles were not shown in a time-dependent manner: gradually up-regulated or gradually down-regulated in MDA-treated group according to each time point. For this reason we focused on commonly deregulated genes in MDA-treated group and we postulated that significantly up- or down-regulated genes could be potential genomic biomarkers for acute hepatotoxicity.

However, microarray analysis using $10 \mathrm{~K}$ mouse oligo chip showed that individual variations among treated group were observed in $k$-means clustering analysis, although liver samples were selected from mice with 
similar histopathological lesions among MDA-treated group. Quality assessment of microarray experiment using box plot and scatter plot represented that there was no significant variation within an array and between arrays (data not shown). Individual variations might be caused by differences of biological or genetic sensitivity for MDA, although common histopathological lesions were shown. The $10 \mathrm{~K}$ mouse oligo chip analysis detected differentially expressed gene sets in MDA-treated group. Among the genes regulated in common at all time points, several genes related to hepatotoxicity including liver hyperplasia/hyperproliferation (Timp1), liver necrosis/cell death (Cd14, Mt1f, Timp1, and Pmap1), liver hemorrhaging (Mt1f), liver cholestasis (Akr1c3, Hpx, and Slc10a2), and liver inflammations (Cd14 and Hpx) were identified using IPA with knowledge-based database. Up-regulation of Timp1 involved in many diseases, including hepatic fibrosis, aortic stenosis, various cancers, and Alzheimer's disease, were identified in MDAtreated group. Timp1 belongs to TIMP gene family which is natural inhibitor of the matrix metalloproteinase (MMPs), is known to promote cell proliferation (Gasson et al., 1985) and also have an anti-apoptotic function (Liu et al., 2005). Regarding hepatotoxicity, it has been known that expression of Timp1 and MMP promotes the liver fibrosis (Fiorucci et al., 2005). In addition, overexpression of Timp1 is known to block the proliferation (Martin et al., 1999) and have anti-apoptotic function in hepatic stellate cells (Murphy et al., 2002). Histopathological observation showed that MDA administration induced severe necrosis of hepatocytes and several genes related to liver necrosis such as Cd14, Mt1f, and Pmaip1 as well as Timp1. Cd14 is a surface protein preferentially expressed on monocytes or macrophages and its apoptotic function in the liver has been reported (Yin et al., 2001). Mt1f has been known to involve in apoptosis, oxidative stress, and cytotoxicity and its necrotic function in liver has been also reported (Masters et al., 1994). Pmaip1 containing Bcl2-homology 3 domain has been known to involve in p53 signaling pathway and plays a role in cytotoxicity and apoptosis in various cell type and conditions (Shibue et al., 2003; Strohecker et al., 2008; Zhang et al., 2008). In present study, regarding necrosis or apoptosis of hepatocytes, Timp1, Cd14, and MtIf were up-regulated, while Pmaip1 was down-regulated in MDA-treated group. On the other hands, several genes related to cholestasis such as Akr1c3, Hpx, and S/c10a2 was also identified. Akr1c3, Hpx, and Slc10a2 have been reported that gene expressions were altered during familiar intrahepatic cholestasis, type 1 or type 2 (Alvarez et al., 2004; Demeilliers et al., 2006). Slc10a2 also plays a role in transporting the bile acid or salts (Dawson et al., 2003) and altered expression of S/c10a2 suggested that transport might be disrupted. In addition to toxicological function, pathway analysis represented that significant pathways such as GABA receptor signaling or hepatic fibrosis or hepatic cell activation were involved in MDAinduced hepatotoxicity. Our data showed potential biomarkers which are mediated after liver damage. The identified genes offer valuable information related to molecular mechanisms of hepatotoxicity. This data may contribute in elucidation of the molecular events during liver injury and in discovery of the potential biomarkers for hepatotoxicity. To confirm and verify the potential biomarkers for hepatotoxicity, extended microarray analyses using different hepatotoxicants including aromatic amine species are necessary. Furthermore, gene expression comparison in the liver after administration of MDA and active metabolite, N-Acetyl MDA, should be necessary to understand the molecular mechanism of MDA-induced hepatotoxicity.

\section{ACKNOWLEDGEMENTS}

This work was supported by the Ministry of Science and Technology as part of the 2005 Project for Developing a Toxicological Evaluation System for Specific Toxicity-inducing Chemicals at the Korea Institute of Toxicology.

\section{REFERENCES}

Alvarez, L., Jara, P., Sánchez-Sabaté, E., Hierro, L., Larrauri, J., Díaz, M.C., Camarena, C., De la Vega, A., Frauca, E., López-Collazo, E. and Lapunzina, P. (2004). Reduced hepatic expression of farnesoid $X$ receptor in hereditary cholestasis associated to mutation in ATP8B1. Hum. Mol. Genet., 13, 2451-2460

Bastian, P.G. (1984). Occupational hepatitis caused by methylenedianiline. Med. J., 141, 533-535.

Dawson, P.A., Haywood, J., Craddock, A.L., Wilson, M., Tietjen, M., Kluckman, K., Maeda, N. and Parks, J.S. (2003). Targeted deletion of the ileal bile acid transporter eliminates enterohepatic cycling of bile acids in mice. $J$. Biol. Chem., 278, 33920-33927.

Demeilliers, C., Jacquemin, E., Barbu, V., Mergey, M., Paye, F., Fouassier, L., Chignard, N., Housset, C. and Lomri, N.E. (2006). Altered hepatobiliary gene expressions in PFIC1: ATP8B1 gene defect is associated with CFTR down regulation. Hepatology, 43, 1125-1134.

Fiorucci, S., Rizzo, G., Antonelli, E., Renga, B., Mencarelli, A., Riccardi, L., Orlandi, S., Pruzanski, M., Morelli, A. and Pellicciari, R. (2005). A farnesoid $x$ receptor-small heterodimer partner regulatory cascade modulates tissue metalloproteinase inhibitor-1 and matrix metalloprotease expression in hepatic stellate cells and promotes resolu- 
tion of liver fibrosis. J. Pharmacol. Exp. Ther., 314, 584595.

Gasson, J.C., Golde, D.W., Kaufman, S.E., Westbrook, C.A., Hewick, R.M., Kaufman, R.J., Wong, G.G., Temple, P.A. Leary, A.C. and Brown, E.L. (1985). Molecular characterization and expression of the gene encoding human erythroid-potentiating activity. Nature, 315, 768-771.

Hamadeh, H.K., Bushel, P.R., Jayadev, S., Martin, K., DiSorbo, O., Sieber, S., Bennett, L., Tennant, R., Stoll, R., Barrett, J.C., Blanchard, K., Paules, R.S. and Afshari, C.A. (2002). Gene expression analysis reveals chemicalspecific profiles. Toxicol. Sci., 67, 219-231.

Heijne, W.H., Slitt, A.L., van Bladeren, P.J., Groten, J.P., Klaassen, C.D., Stierum, R.H. and van Ommen, B. (2004). Bromobenzene-induced hepatotoxicity at the transcriptome level. Toxicol. Sci., 79, 411-422.

Huang, Q., Jin, X., Gaillard, E.T., Knight, B.L., Pack, F.D., Stoltz, J.H., Jayadev, S. and Blanchard, K.T. (2004). Gene expression profiling reveals multiple toxicity endpoints induced by hepatotoxicants. Mutat. Res., 549, 147-167.

Liu, X.W., Taube, M.E., Jung, K.K., Dong, Z., Lee, Y.J., Roshy, S., Sloane, B.F., Fridman, R. and Kim, H.R. (2005). Tissue inhibitor of metalloproteinase-1 protects human breast epithelial cells from extrinsic cell death: a potential oncogenic activity of tissue inhibitor of metalloproteinase-1. Cancer Res., 65, 898-906.

Martelli, A., Carrozzino, R., Mattioli, F. and Brambilla, G. (2002). DNA damage induced by 4,4'-methylenedianiline in primary cultures of hepatocytes and thyreocytes from rats and humans. Toxicol. Appl. Pharmacol., 182, 219225.

Martin, D.C., Fowlkes, J.L., Babic, B. and Khokha, R. (1999). Insulin-like growth factor II signaling in neoplastic proliferation is blocked by transgenic expression of the metalloproteinase inhibitor TIMP-1. J. Cell. Biol., 146, 881-892.

Masters, B.A., Kelly, E.J., Quaife, C.J., Brinster, R.L. and Palmiter, R.D. (1994). Targeted disruption of metallothionein I and II genes increases sensitivity to cadmium. Proc. Natl. Acad. Sci. U.S.A., 91, 584-588.

McQueen, C.A. and Williams, G.M. (1990). Review of the genotoxicity and carcinogenicity of 4,4'-methylene-dianiline and 4,4'-methylene-bis-2-chloroaniline. Mutat. Res., 239, 133-142.

Murphy, F.R., Issa, R., Zhou, X., Ratnarajah, S., Nagase, H., Arthur, M.J., Benyon, C. and Iredale, J.P. (2002). Inhibition of apoptosis of activated hepatic stellate cells by tissue inhibitor of metalloproteinase-1 is mediated via effects on matrix metalloproteinase inhibition: implications for reversibility of liver fibrosis. J. Biol. Chem., 277, 1106911076.

Nichols, L. (2004). The Epping Jaundice outbreak: mortality after 38 years of follow-up. Int. Arch. Occup. Environ. Health, 77, 592-594.
Oh, J.H., Park, H.J., Hwang, J.Y., Jeong, S.Y., Lim, J.S., Kim. Y.B. and Yoon, S. (2007). Gene expression analysis of hepatic response induced by gentamicin in mice. Mol. Cell. Toxicol., 3, 60-67.

Oh, J.H., Yang, M.J., Yang, Y.S., Park, H.J., Heo, S.H., Lee, E.H., Song, C.W. and Yoon, S. (2009a). Microarray-based analysis of the lung recovery process after stainless steel welding fume exposure in Sprague-Dawley rats. Inhal. Tox., 21, 1-27.

Oh, J.H., Oh, M.J., Park, H.J., Kim, S.J., Park, S.M., Yoon, H.J., Cho, J.W. and Yoon, S. (2009b). Analysis of gene expression in the testes of mice exposed to bisphenol $A$ and nonylphenol. Biochip J., in press.

Oh, J.H., Park, H.J., Jeong, S.Y., Lim, J.S., Hwang, J.Y., Kim. Y.B. and Yoon, S. (2006). Gene expression profiling of early renal toxicity induced by gentamicin in mice. Mol. Cell. Toxicol., 2, 185-192.

Robert, A., Ducos, P. and Francin, J.M. (1995). Determination of urinary 4,4'-methylenedianiline and its acetylated metabolites by solid-phase extraction and HPLC analysis with UV and electrochemical detection. Int. Arch. Occup. Environ. Health, 68, 44-51

Santa Cruz, V., Liu, H., Kaphalia, L. and Kanz, M.F. (2007). Effects of methylenedianiline on tight junction permeability of biliary epithelial cells in vivo and in vitro. Toxicol. Lett., 169, 13-25.

Schutze, D., Sepai, O., Lewalter, J., Miksche, L., Henschler, D. and Sabbioni, G. (1995). Biomonitoring of workers exposed to 4,4'-methylenedianiline or 4,4'-methylenediphenyl diisocyanate. Carcinogenesis, 16, 573-582.

Shibue, T., Takeda, K., Oda, E., Tanaka, H., Murasawa, H., Takaoka, A., Morishita, Y., Akira, S., Taniguchi, T. and Tanaka, N. (2003). Integral role of Noxa in p53-mediated apoptotic response. Genes Dev., 17, 2233-2238.

Strohecker, A.M., Yehiely, F., Chen, F. and Cryns, V.L. (2008). Caspase cleavage of HER-2 releases a bad-like cell death effector. J. Biol. Chem., 283, 18269-18282.

Yin, M., Bradford, B.U., Wheeler, M.D., Uesugi, T., Froh, M., Goyert, S.M. and Thurman, R.G. (2001). Reduced early alcohol-induced liver injury in CD14-deficient mice. $J$. Immunol., 166, 4737-4742.

Zhang, X., Lambert, J.C., Doll, M.A., Walraven, J.M., Arteel, G.E. and Hein, D.W. (2006). 4,4'-methylenedianilineinduced hepatotoxicity is modified by $\mathrm{N}$-acetyltransferase 2 (NAT2) acetylator polymorphism in the rat. J. Pharmacol. Exp. Ther., 316, 289-294.

Zhang, G., Park, M.A., Mitchell, C., Walker, T., Hamed, H., Studer, E., Graf, M., Rahmani, M., Gupta, S., Hylemon, P.B., Fisher, P.B., Grant, S. and Dent, P. (2008). Multiple cyclin kinase inhibitors promote bile acid-induced apoptosis and autophagy in primary hepatocytes via p53-CD95dependent signaling. J. Biol. Chem., 283, 24343-24358. 